\title{
Patienten mit venösen Thrombembolien sind heterogen: Daten aus dem GARFIELD-VTE-Register
}

Ageno Walter et al. Characteristics and Management of Patients with Venous Thromboembolism: The GARFIELD-VTE Registry. Thromb Haemost. 2019 Feb; 119 (2): 319-327

Tiefe Venenthrombosen (TVT) und Lungenarterienembolien (LAE) verursachen beträchtliche Morbidität und Mortalität mit mehr als 500000 Todesfällen in der europäischen Union jedes Jahr. Die Ätiologie ist multifaktoriell. Auch das Management von venösen Thrombembolien, die TVT und LAE umfassen, variiert weltweit.

Eine Gruppe von Autoren um Ageno aus Varese untersuchte in einer prospektiven Observationsstudie die Charakteristika und das Management von Patienten mit venösen Thrombembolien. In die GARFIELDVTE-Studie (Global Anticoagulant Registry in the FIELD - Venous Thromboembolism) wurden von Mai 2015 bis Januar 2017 in 417 Kliniken in 28 Ländern 10685 Patienten mit diagnostizierten venösen Thrombembolien eingeschlossen. Alle Patienten wurden über 3 Jahre nachbeobachtet. In der aktuellen Arbeit beschreiben die Autoren die Grundcharakteristika der Studienpopulation und ihr Management innerhalb der ersten 30 Tage nach Diagnose.
Ergebnisse:

Das mediane Alter lag bei 60,2 Jahren, der Anteil der männlichen Patienten lag bei $50,4 \%, 61,7 \%$ hatten eine TVT, 38,3\% eine LAE mit oder ohne TVT. 32,3\% waren adipös (Body Mass Index $\geq 30 \mathrm{~kg} / \mathrm{m} 2$ ). Der häufigste Risikofaktor war Operation (12,5\%), Hospitalisierung $(12,0 \%)$ und Trauma an den unteren Extremitäten (7,8\%). Zum Zeitpunkt der Diagnosestellung hatten $10,1 \%$ eine aktive Krebserkrankung und $5,7 \%$ waren chronisch immobilisiert. Die Behandlung war eine Antikoagulantientherapie alleine bei $90,9 \%$ der Patienten, 5,1\% erhielten eine thrombolytische und/oder chirurgische/mechanische Therapie \pm Antikoagulation und $4,0 \%$ erhielten keine Therapie. Vor der Diagnose erhielten $12,8 \%$ der Patienten eine Antikoagulantientherapie alleine und $0,2 \%$ eine thrombolytische und/oder chirurgische/mechanische Therapie \pm Antikoagulation. Nach der Diagnose wurde eine Antikoagulantientherapie alleine bei $17,6 \%$, verabreicht, sie war gefolgt von einer direkten oralen Antikoagulantientherapie bei $16,4 \%$ oder der Gabe von Vitamin-K-Antagonisten bei 26,8\%. Direkte orale Antikoagulation wurde verschrieben bei $32,3 \%$ der Patienten, während $5,9 \%$ alleine Vitamin-K-Antagonisten erhielten.

\section{FAZIT}

Die Daten zeigen, so die Autoren, dass es substanzielle Heterogenität bei der Behandlung von Patienten mit venösen Thrombembolien gibt. Diese Heterogenität kann einerseits durch die unterschiedlichen Patienten-Charakteristika erklärt werden, die die Notwendigkeit für eine individualisierte therapeutische Herangehensweise unterstreichen, andererseits durch Unterschiede in den Gesundheitssystemen in den verschiedenen Ländern. Der Effekt der verschiedenen Strategien wird in den weiteren Follow-up-Daten nachvollziehbar werden. Die Autoren sehen die hauptsächlichen Limitationen ihrer Studie in der Erhebung von nichtrandomisierten Daten und dem zu Grunde liegenden Selektionsbias.

MOR Dr. med. Benedikt Lampl, Regensburg 